\title{
The effect of transient temporal pulse shape on surface temperature and tungsten damage
}

\author{
J.H. Yu ${ }^{\mathrm{a}}$, G. De Temmerman ${ }^{\mathrm{b}}$, R.P. Doerner ${ }^{\mathrm{a}}$, R.A. Pitts ${ }^{\mathrm{b}}$, and M.A. van den Berg \\ aUniversity of California, San Diego, La Jolla, CA 92093-0417, USA \\ 'ITER Organization, Route de Vinon sur Verdon, CS90 046, 13067 St-Paul-Lez-Durance Cedex, France \\ 'FOM DIFFER, Dutch Institute for Fundamental Energy Research, Association EURATOM-FOM, \\ Netherlands \\ Email: j2yu@eng.ucsd.edu
}

\begin{abstract}
The plasma-facing components (PFCs) in future fusion devices such as ITER will receive intense transient heat fluxes from plasma instabilities, such as Edge Localized Modes (ELMs) and disruptions, which will limit material lifetime. The energy density and transient pulse duration are typically used to characterize the PFC damage threshold. However, these parameters are not sufficient to define a damage threshold, because different transient pulse shapes with the same energy density and same pulse duration produce different peak surface temperatures (and thus stresses, which ultimately determine material damage). The surface temperature and damage of tungsten (the material to be used for the ITER divertor target armor) in the form of surface roughening and melting are investigated using various temporal pulse shapes from an Nd:YAG laser in the PISCES-B facility. The heat flux factor is examined and shown to be an inadequate parameter to characterize the temperature rise except for square temporal pulse shapes. For ELM-like heat pulses, the long tail in the temporal shape results in a lower peak surface temperature and less damage compared to a symmetric triangle pulse with equal energy density.
\end{abstract}

\section{Introduction}

Transient heat loads due to plasma instabilities such as Edge Localized Modes (ELMs) and plasma disruptions are expected to play a major role in limiting the lifetime of plasma-facing components (PFCs). Specifying allowable heat load limits is an important task for the design of mitigation systems such as magnetic perturbation coils, which will be used in ITER to reduce the ELM energy density while increasing the ELM frequency. The present ITER specification for the maximum allowable ELM energy loss from the plasma [1] is based on the energy density of heat pulses which caused melting of tungsten (W) targets exposed to intense plasma pulses in the QSPA-T test facility [2]. ITER will begin operations with a full-W armored divertor [3]. In the case of the QSPA-T plasma pulses, the rise time of their 
temporal waveform is quite similar to that of real ELM pulse shapes observed in current tokamaks at divertor targerts [4]. However, most electron-beam laboratory tests of the response of W surfaces to multiple rapid transients have used square wave pulse shapes (see e.g. [5]).The temporal pulse shape affects the peak surface temperature reached during the pulse, and thus affects the strain rate which is known to influence the ductility of a material [6]. We show that with the same energy density, different pulse shapes result in different peak surface temperatures and different levels of material damage.

Ultimately the goal is to better understand how realistic pulse shapes from plasma instabilities affect the established damage threshold for PFCs in ITER. This work also provides guidance for comparing transient heat testing results across different devices with different pulse shapes.

\section{Experimental Setup}

Rolled commercial grade polycrystalline W with purity $>99.97 \%$ from Plansee was cut into discs (25.4 mm diameter and $2 \mathrm{~mm}$ thick) using electric discharge machining. The as-received polycrystalline $\mathrm{W}$ had elongated grains with size 1 to $2 \mu \mathrm{m}$ wide and 10 to $100 \mu \mathrm{m}$ long with grain orientation parallel to the sample surface. Samples were surface ground with successively finer grades of abrasive from 220 to 1200 grit on a Struers TegraPol-11 grinding and polishing machine, and then polished with successively finer grades of polishing paste ranging from 9 to $1 \mu \mathrm{m}$. The root-mean-squared surface roughness with no laser irradiation was $R_{r m s}=0.05 \pm 0.02 \mu \mathrm{m}$ as measured with a confocal microscope using line profiles. Samples were then ultra-sonically cleaned in acetone followed by ethanol before laser exposure.

An Nd:YAG laser with maximum peak power of $5 \mathrm{~kW}$ with wavelength of $1064 \mathrm{~nm}$ was used to create various temporal heating pulses on $\mathrm{W}$ samples mounted on the sample manipulator in the PISCESB linear plasma device [7]. The laser was guided by a set of turning mirrors over a distance of $\sim 14 \mathrm{~m}$ and a lens focused the beam on the target to obtain the desired spot size. The laser was incident on the target at an angle of 15 degrees with respect to normal, resulting in a slight asymmetry of the 2D distribution of laser intensity on the target surface. For the majority of the data in this paper the base temperature of the $\mathrm{W}$ was room temperature and the sample was not exposed to plasma. The laser produced square, triangular, and ELM-like temporal pulse shapes and the pulse width was varied from 0.6 to $10 \mathrm{~ms}$. For each pulse shape, 100 laser pulses were fired on the same location on the target with a repetition rate of $0.2 \mathrm{~Hz}$ and laser spot size of $0.5 \mathrm{~mm}$ diameter. Triangular pulse shapes with three different rise times were compared with two types of square pulses, and all five pulse shapes had approximately the same energy density. We also investigated the subtleties of ELM-shaped heat pulses. The laser was moved to a new location on the target for each pulse shape, and the spatial profile of the laser intensity was measured at the output of the laser to be approximately Gaussian. 
The surface temperature of the $\mathrm{W}$ target was measured during the laser pulse using a two-color fast pyrometer [8] with temporal resolution of $\sim 10 \mu$ s. For the experiments presented here, the pyrometer detected thermal emission at 1300 and $1550 \mathrm{~nm}$ wavelengths using interference filters with bandwidths of $100 \mathrm{~nm}$. An absolute intensity technique and a two-color ratio technique were used to analyze pyrometer data for the surface temperature measurement. The absolute intensity technique provided better signal to noise and is presented here. It relies on knowledge of the spot size because the pyrometer viewing area (diameter $=2 \mathrm{~cm}$ ) was much larger than the laser spot (so that multiple laser spots on the target could be viewed with a fixed pyrometer alignment). The laser spot size was measured with a confocal microscope after 100 laser heating cycles by identifying the edge of the roughened region on the $\mathrm{W}$ surface when the central region of the spot began to melt. For all loading conditions an average spot size of $0.5 \mathrm{~mm}$ diameter was used, and uncertainty in spot size is the largest source of error in estimating the energy density and temperature.

The temperature calibration was performed with steady-state plasma-heated W samples over a range of sample temperatures measured with a thermocouple. The small steady-state signal offset due to reflected near-infrared signal from the hot cathode was corrected for by turning off the plasma discharge but leaving the cathode hot. The error in the temperature measurement was minimized by comparing the absolute intensity method to the two-color ratio technique, which is insensitive to laser and pyrometer measurement spot sizes, and insensitive to the surface emissivity. The calibration factor at each wavelength was adjusted so that the peak temperature from the absolute intensity method agreed with the two-color ratio technique, with the constraint of keeping the ratio of calibration factors constant. All inputs to the absolute intensity pyrometer analysis (laser and pyrometer spot sizes, and the calibration factors) were then fixed for each sample, so that the temperature measurements were self-consistent. The absolute intensity analysis used here to determine the surface temperature assumes that all the thermal radiation is emitted uniformly from the entire laser spot, and thus the calculation gives an average temperature for the spot.

A photodiode detected the laser intensity as a function of time in order to measure the temporal pulse shape of the heat pulse to the target. The photodiode was located near the laser and detected a small fraction of laser light scattered from a turning mirror.

\section{Results and Discussion}

\subsection{Temperature measurements}


Figures 1(a) and (b) show the absorbed power density delivered by the laser and the measured surface temperature for five different pulse shapes. All had the same energy density (1.8 $\left.\mathrm{MJm}^{-2}\right)$ to within $3 \%$. The absorbed laser power was calculated by comparing the integral of the photodiode signal to the measured laser pulse energy, and taking into account the spot size on the target, estimated beamline losses, and a W reflectivity of 50\% [8, 9]. The photodiode measurement occurred simultaneously with the $\mathrm{W}$ target irradiation and pyrometer measurement. The change in the reflectivity of $\mathrm{W}$ due to laser heating [10] was not taken into account. The surface temperature measured with a fast pyrometer is plotted only for $\mathrm{T}>1000 \mathrm{~K}$ because the pyrometer required a minimum temperature in order to detect thermal emission from the relatively small laser spot size of $\sim 0.5 \mathrm{~mm}$.

The results show clearly that the peak surface temperature depends on the pulse shape, even with the same energy density in the heating pulses. It is worth noting that four of the pulse shapes have approximately the same peak power (in addition to the same energy density), but the peak surface temperatures are different. The long square pulse ("half-amplitude square"), shown as the dashed line, produced the lowest peak surface temperature of all 5 pulses. The short square pulse ("half-width square”) shown with dot-dashed lines in figure 1 was half the duration compared to the other pulses and resulted in the highest peak surface temperature. Three triangular pulses with different rise times created progressively higher peak surface temperatures as the rise time was increased. We denote the triangular pulse shapes as follows: the pulse with the shortest rise time is called a "negative ramp”, the pulse with equal rise and fall times is called a "symmetric triangle", and the longest rise time pulse is called a “positive ramp.” For the same energy density, the peak surface temperatures reached by the triangular pulses are bracketed by those from the half-amplitude square pulse on the low side and the half-width square pulse on the high side. 

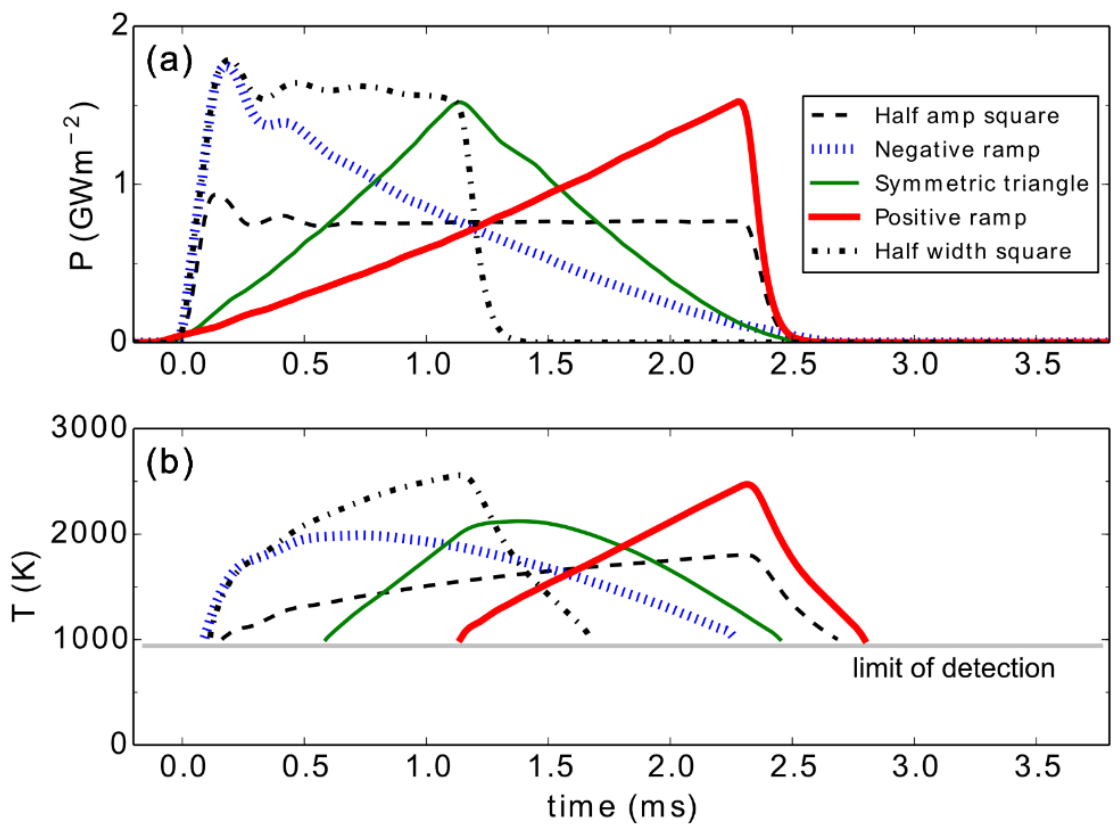

Figure 1 (a) The absorbed power densities and (b) the spatially-averaged temperature measurements for 5 pulse shapes with approximately equal energy density of $1.8 \mathrm{MJm}^{-2}$ : half-amplitude square (dashed line with $\mathrm{T}_{\text {peak }}=1800$ $\mathrm{K}$ ), half-width square (dot-dashed line with $\mathrm{T}_{\text {peak }}=2560 \mathrm{~K}$ ), negative ramp triangle (blue hashed line with $\mathrm{T}_{\text {peak }}=$ $1980 \mathrm{~K}$ ), symmetric triangle (green solid line with $\mathrm{T}_{\text {peak }}=2120 \mathrm{~K}$ ), positive ramp triangle (thick red line with $\mathrm{T}_{\text {peak }}=$ $2490 \mathrm{~K}$ ). Each measurement is an average of 4 laser shots taken approximately midway through the 100 pulse heating cycle. All measurements were taken from the same sample.

\subsection{Analytic solutions to the heat equation}

The surface temperature rise due to different heat pulses can be written analytically by solving the 1D heat equation. For a square pulse with pulse duration $\tau_{s}$, the peak surface temperature rise is given by

$$
\Delta T_{s}=2 P_{o} \tau_{s}^{1 / 2}(\pi \kappa \rho c)^{-1 / 2}
$$

where $P_{o}$ is the peak power density in units of $\mathrm{Wm}^{-2}, \kappa$ is the thermal conductivity $\left(\mathrm{W} \mathrm{m}^{-1} \mathrm{~K}^{-1}\right), \rho$ is the density $\left(\mathrm{kg} \mathrm{m}^{-3}\right), c$ is the specific heat $\left(\mathrm{J} \mathrm{kg}^{-1} \mathrm{~K}^{-1}\right)$, and the subscript $s$ denotes square pulse. The $1 \mathrm{D}$ heat equation can also be solved for a triangular pulse with arbitrary rise time [11], and the peak surface temperature rise $\Delta T_{t}$ can be normalized to $\Delta T_{s}$ as follows if the triangular and square pulses have equal energy density and peak power:

$$
\frac{\Delta T_{t}}{\Delta T_{S}}=\frac{4}{3}\left(4-\frac{\tau_{r}}{\tau_{s}}\right)^{-1 / 2}
$$

where $\tau_{r}$ is the rise time of the triangular pulse. 
Results from 3D finite element simulations using ANSYS are shown as red circles in figure 2 and match the analytic solution. The simulation used a spatially uniform heat flux in a circular area. The simulation included radial heat transport and the temperature dependence of the thermomechanical properties of W. Since the analytic solution assumes that the thermomechanical properties do not change with temperature and also ignores radial heat diffusion, the analytic solution should not be used in place of simulations when absolute values for temperature are desired, despite the excellent agreement in figure 2. This is because normalizing the peak temperature rise to that from a square pulse eliminates the dependence on thermomechanical properties and also reduces radial heat transfer effects, because any radial heat transfer would occur in a similar way for both the triangular and square pulse shapes. An additional advantage of normalizing in this way is that $\Delta T_{t}$ as a function of rise time can be easily compared for a variety of energy densities and pulse widths, and the relative peak temperature rise values for square and triangular pulses can be seen immediately.

The $1 \mathrm{D}$ solution predicts that a positive ramp triangular pulse with $\tau_{r}=2 \tau_{s}$ has a peak surface temperature a factor $\sqrt{2}$ higher than that for a negative ramp pulse $\left(\tau_{r}=0\right)$. The measured peak surface temperature rise for three triangular pulse shapes, normalized to a half-width square pulse, agrees reasonably well with the $1 \mathrm{D}$ solution, particularly the trend of the data points (the data set with $\tau_{s}=2.5$ ms was normalized to the $1 \mathrm{D}$ solution for a symmetric triangular pulse at $\frac{\tau_{r}}{\tau_{s}}=1$ because there was no half-width square pulse data for that case). Most data points lie above the analytic solution because the measured temperature rise from the half width square pulse used to normalize the data was lower than expected compared to a perfect square pulse: the slight overshoot at the beginning of the laser square pulse caused a lower flat-top power (and thus lower peak temperature) in order to match the energy density to the other pulse shapes.

The non-normalized solution for the peak surface temperature rise for a triangular pulse shape is

$$
\Delta T_{t}=\frac{4}{3} \frac{P_{o} \tau_{t}}{\sqrt{\pi \kappa \rho c\left(2 \tau_{t}-\tau_{r}\right)}}
$$

where $\tau_{t}$ is the total pulse width and the other quantities are defined above. This solution can also be written in terms of the total absorbed energy density $E$, because for a triangular pulse $E=\frac{1}{2} P_{o} \tau_{t}$. When Eq. 3 is compared to the ANSYS simulations, we find that the peak temperature rise predicted by ANSYS is higher than that computed from the analytic solution. The discrepancy can be significant; for example, using a symmetric triangle pulse shape with $P_{o}=2 \mathrm{GWm}^{-2}, \tau_{t}=10^{-3} \mathrm{~s}$, and heating diameter of $1.5 \mathrm{~mm}$, ANSYS predicts a peak temperature rise $\left(\Delta T_{t}=2172 \mathrm{~K}\right)$ that is larger by $333 \mathrm{~K}$ compared with the $1 \mathrm{D}$ heat equation solution with constant room-temperature values for $\mathrm{W}$ material properties. The discrepancy 
is mainly caused by the temperature dependence of $\kappa$, which for $\mathrm{W}$ has a lower value at higher temperature. Using the same symmetric triangle heating pulse in a simulation with constant material properties (no temperature dependence) and comparing to the 1D solution, it was found that radial heat diffusion significantly affected the peak central temperature when the spot size diameter was $0.5 \mathrm{~mm}$, but not in the $1.5 \mathrm{~mm}$ case.

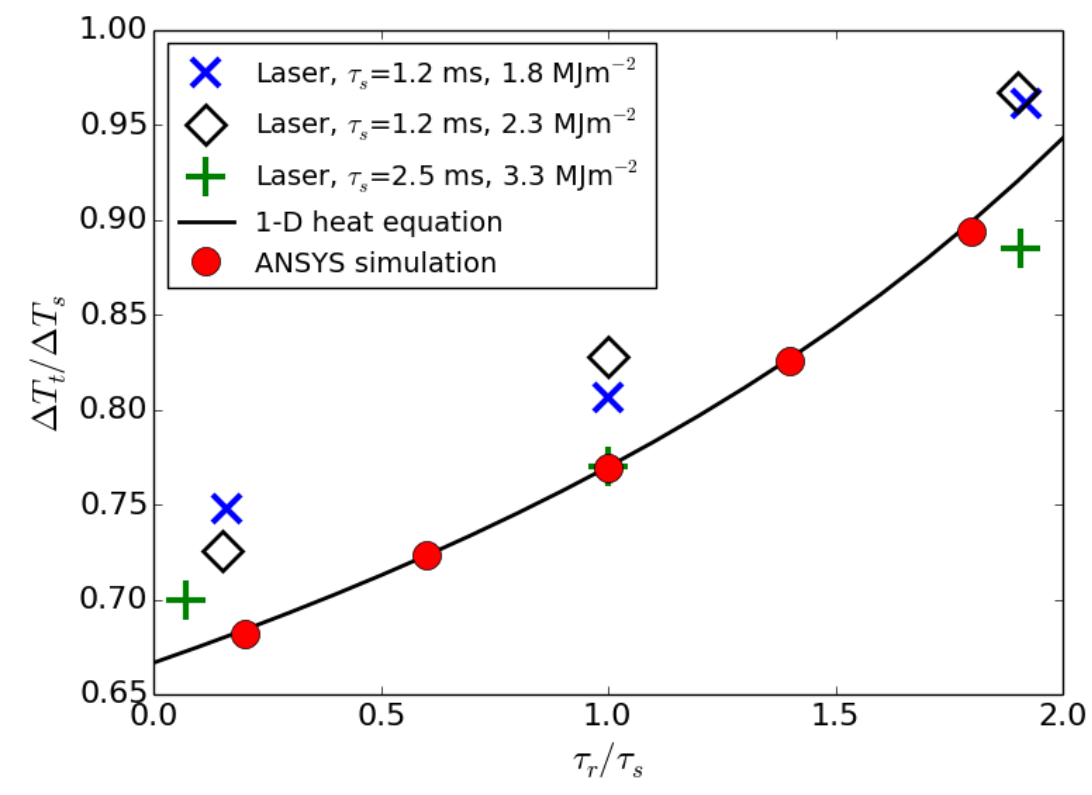

Figure 2 The peak surface temperature rise $\Delta T_{t}$ for triangular shaped heating pulses as a function of the heating pulse rise time $\tau_{r} . \Delta T_{t}$ is normalized to the peak temperature rise $\Delta T_{s}$ for a square pulse with the same energy density and same peak power. The x-axis is normalized to the pulse width $\tau_{s}$ of the square pulse. Three sets of laser data are shown as the "x," diamond, and "+" data points (2.5 ms data set was normalized to the heat equation solution for $\frac{\tau_{r}}{\tau_{s}}=1$ ). Black line is Eq. 2, and red circles show results from ANSYS modeling.

\subsection{Images of W surface after laser heating}

A confocal microscope was used to image the laser-irradiated $\mathrm{W}$ samples following exposure. The images in figure 3(a) - 3(e) show the W surface after 100 heating pulses from each of the five pulse shapes shown in figure 1 (two square pulses and three triangular pulses with different rise times), all with the same absorbed energy density of $1.8 \mathrm{MJm}^{-2}$ (see figure 1). Figures 3(f) - (j) show laser spots from the same five different pulse shapes but now with an increased absorbed energy density of $2.3 \mathrm{MJm}^{-2}$. Each row of images is ordered in terms of increasing damage, and the melting threshold near the center of the spot is traversed for each energy density. Surface imperfections due to the polishing process appear as lines in images such as figure 3(c). In common with other transient heat experiments [5, 12, 13, 14], 
recrystallization and grain growth is observed and $\mathrm{W}$ becomes damaged in the form of surface roughening after multiple heating cycles. At sufficiently high temperatures, the center of the laser spot melts. The series of images shows that the level of damage varies significantly, ranging from surface roughening to the formation of a melt crater, even though the energy density in each set of images is constant. The damage is observed to be correlated with the peak surface temperature reached during the pulse.

The pulse shape that yields the lowest observed damage and lowest measured temperature is the half-amplitude, full-duration square heat pulse shown in figures 3(a) and (f). The 1D heat equation solutions in equations 1 and 2 actually predict that the triangular negative ramp pulse shape produces a slightly lower $\Delta T$ than the half-amplitude square pulse with the same energy density $\left(\frac{\Delta T \text { (neg ramp })}{\Delta T \text { (half amplitude square })}=0.94\right)$. Possible causes for the discrepancy between this prediction and the laser data are 2D heat transport due to the small laser spot (which is on the same order as the heat penetration depth), and the overshoot at the beginning of the laser pulse. The 1D solutions also predict that a triangular pulse will produce the same peak $\Delta T$ as that from a half-amplitude square pulse with the same energy density when the rise time is $\tau_{r} / \tau_{t}=0.22$.

In the $1.8 \mathrm{MJm}^{-2}$ data set, the onset of melting near the center of the spot is observed for the symmetric triangle pulse shape in figure 3(c), while in the $2.3 \mathrm{MJm}^{-2}$ data set the central region is melted for the negative ramp pulse shape in figure $3(\mathrm{~g})$. The central melt regions become progressively larger moving to the right hand side of figure 3. Compared to the other triangular pulses, the positive ramp triangular pulses in (d) and (i) create the highest level of damage. In figure 3(e) and (j) laser spots are shown after heating due to square pulses with the same peak power as the triangular pulses but with onehalf the duration. This full-amplitude, half-width square pulse produces the highest level of damage and the highest average temperature compared with the other pulse shapes.

Note that the temperature measured by the pyrometer at the onset of melting (figure 3(g), for example) is lower than the $\mathrm{W}$ melting temperature (3695 K). Due to the spatial profile of the laser beam, which was measured to be approximately Gaussian, the center of the spot receives the highest heat flux and presumably reaches a higher temperature than the average temperature reported here. It is therefore difficult to determine if sub-threshold melting occurs in our experiments. We have, however, observed that the size of the melted area increases with number of laser pulses, suggesting that repeated laser pulses cause sub-threshold melting or possibly a decrease in the thermal conductivity. Interestingly, in [10] the temperature was measured with an IR camera and the onset of melting was observed to be $\sim 2770 \mathrm{~K}$ at the center of the laser spot, which is lower than the W melting threshold. The authors attributed this to surface 
roughening causing locally protruding grains which have lower heat dissipation than the bulk material, leading to local melting presumably on a spatial scale below the spatial resolution of the IR camera.

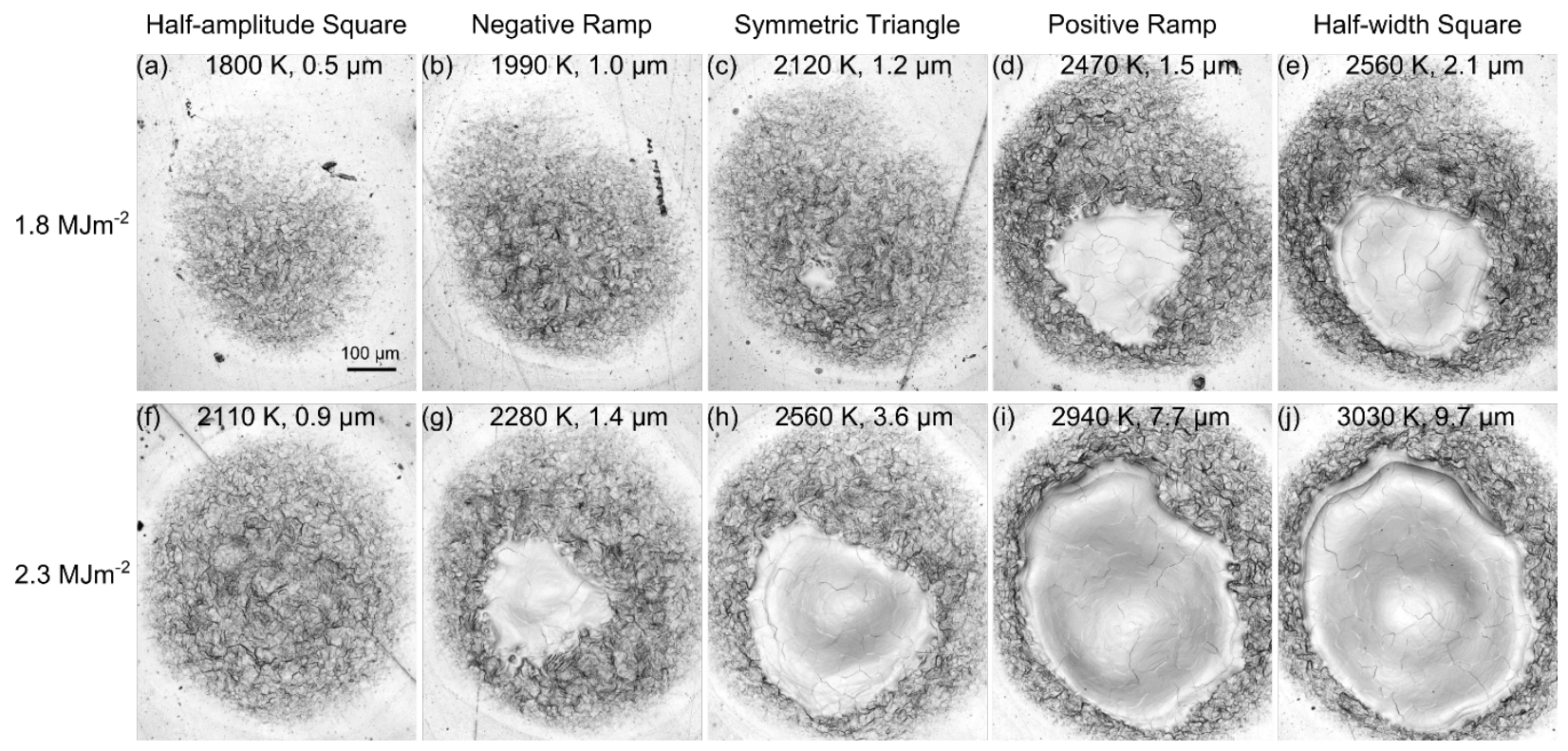

Figure 3 Confocal microsope images after 100 heat pulses. Images in each row show spots exposed to the same absorbed energy density, and different pulse shapes are shown in each column. Figures (a) - (e) correspond to the measurements shown in figure 1. The pulse widths were $2.5 \mathrm{~ms}$ except for (e) and (j) which were $1.25 \mathrm{~ms}$. The peak surface temperature reached during a heating pulse (average measurement from a laser spot diameter of $0.5 \mathrm{~mm}$ ) and the rms surface roughness are shown at the top of each image. Each image is a different location on the same sample.

\subsection{Applicability of Heat Flux Factor}

The heat flux factor $F_{H F}$ has been used rather widely in the fusion community to compare transient heat pulses from different test facilities including electron beams [15] and lasers [12, 13], and has also been used to estimate heat loads on the ITER divertor during unmitigated disruptions [3]. The motivation for defining a "heat flux factor" as $F_{H F}=P \sqrt{t}$ or $F_{H F}=E / \sqrt{t}$, where $P$ and $E$ are the power density and energy density, respectively, is clear from equation 1 , because $F_{H F}$ is directly proportional to $\Delta T$. However, equation 1 is only valid for constant power density (i.e., square pulses), and therefore $F_{H F}$ should not be used for heat pulses with non-square pulse shapes. 
To emphasize this point, figure 4 shows the spatially-averaged temperature rise due to different pulse shapes plotted versus $F_{H F}$. Data points for square pulse shapes are shown as black squares and exhibit a roughly linear increase in $\Delta T$ with $F_{H F}$ up to approximately $25 \mathrm{MJm}^{-2} \mathrm{~s}^{-1 / 2}$. At this spatiallyaveraged $F_{H F}$ the samples begin to melt at the center of the laser spot due to the non-uniform spatial profile of the laser beam. The change in scaling of $\Delta T$ with $F_{H F}$ after the onset of melting is because the measured temperature represents an average temperature over the entire spot, and evaporative cooling becomes a factor when $\mathrm{W}$ is in the liquid phase. As the melted area becomes larger with increasing $F_{H F}$, the average temperature measured for the spot increases and approaches the $\mathrm{W}$ melting temperature when the melted area becomes equal to the entire laser spot. For the triangular pulses in figure 4, the energy density and the full width (entire pulse duration) was used in the calculation of $F_{H F}$.

Figure 4 shows that attempting to define a $F_{H F}$ for triangular pulse shapes is not useful because there is significantly more scatter in the data with triangular pulse shapes included compared to that with square pulses alone. $\Delta T$ for the triangular pulses is generally higher than the square pulses at a given $F_{H F}$ when $F_{H F}$ is defined in terms of energy density. The opposite is true when $F_{H F}$ is defined in terms of the peak power density. The lack of correlation between $\Delta T$ and $F_{H F}$ for all pulse shapes is not surprising because the temperature rise scales as $\sqrt{t}$ only for constant power loads, i.e., square pules. Therefore, the use of $F_{H F}$ is invalid for all pulse shapes except square pulses.

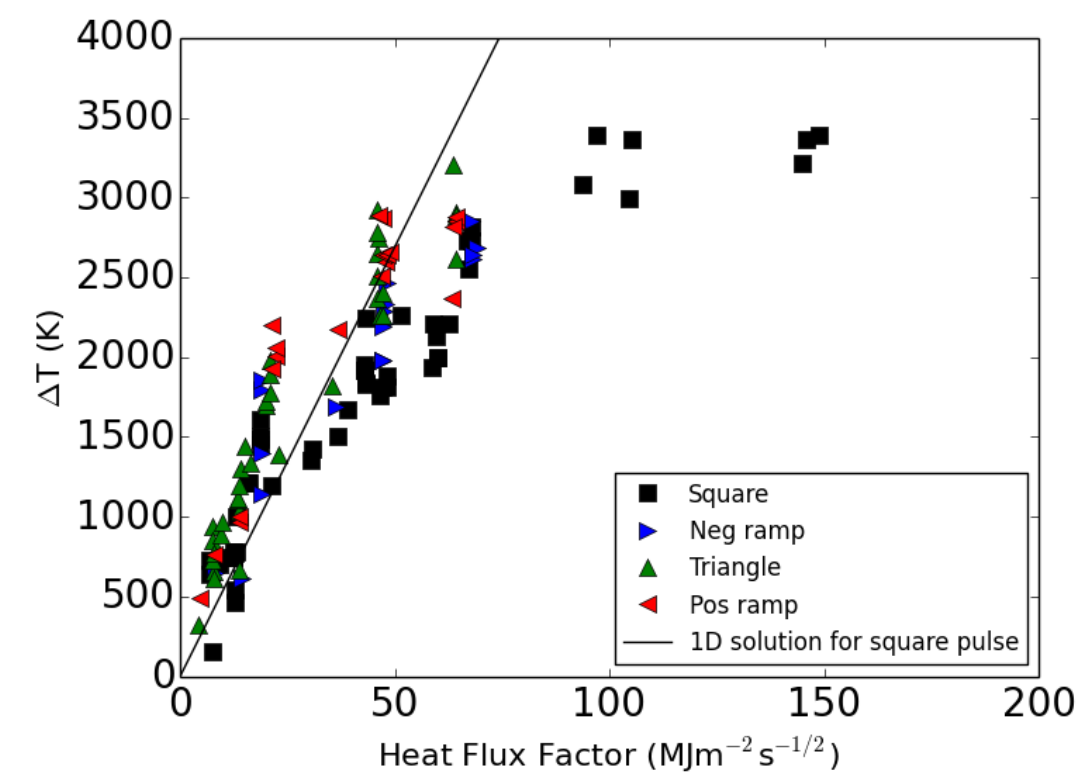

Figure 4 The surface temperature rise $\Delta T$ versus the heat flux factor for various temporal pulse shapes. For the triangular pulse shapes, $F_{H F}$ is defined here in terms of the energy density in the pulse. 
In spite of the fact that $F_{H F}$ is only valid for square pulses, it is worthwhile to look at how $F_{H F}$ is applied to other pulse shapes such as triangular due to the historical use of $F_{H F}$ in the fusion community. There is ambiguity in how to define $F_{H F}$ for non-square pulses because $F_{H F}$ can be defined either in terms of power density or energy density (naturally for square pulses the value of $F_{H F}$ is the same for either definition). For a triangular pulse with any rise time, $F_{H F}$ defined using the peak power density $P_{o}$ reached during the pulse is a factor of 2 larger than $F_{H F}$ defined using the energy density $E$, because for triangular pulses $E=\frac{1}{2} P_{o} \tau$, where $\tau$ is the total pulse duration. Table 1 summarizes the relative $F_{H F}$ values for different temporal pulse shapes with the same energy density, using different definitions of $F_{H F}$.

Table 1 Relative values for the heat flux factor for square and triangular temporal pulse shapes, all with the same energy density $E$. Values are normalized to a half-width square pulse.

\begin{tabular}{|l|c|c|c|}
\hline & Half-width square & Half-amplitude square & Triangle \\
\hline Energy, $F_{H F}=\frac{E}{\sqrt{\tau}}$ & 1 & $\sqrt{2} / 2$ & $\sqrt{2} / 2$ \\
\hline Peak power, $F_{H F}=P_{o} \sqrt{\tau}$ & 1 & $\sqrt{2} / 2$ & $\sqrt{2}$ \\
\hline
\end{tabular}

\subsection{Damage due to transient heating}

One of the principal motivations for the present work is to evaluate the damage threshold for plasmafacing materials exposed to transient heat loads in fusion devices. This raises the question: what is the most important parameter that affects material damage? Is it energy density, peak heat flux, the heat flux factor, or something else? The energy density cannot be the defining parameter for damage because, as we have shown above, different pulse shapes create different peak surface temperatures, even with the same energy density and same pulse duration. In addition, we have already discussed that $F_{H F}$ is only valid for square pulses because for all other pulse shapes $F_{H F}$ does not accurately represent $\Delta T$. Thus, $F_{H F}$ is not adequate to define a damage threshold for arbitrary pulse shapes.

Figure 5 shows that the root-mean-squared surface roughness $R_{r m s}$ is poorly correlated with $F_{H F}$. The surface roughness was measured along line profiles through the center of the laser spot extending to the edge, and typically four measurements were performed at different orientations for each laser spot. The roughness is used as a measure of surface damage because it is a precursor to more extreme damage such as melting and cracking. Roughness occurs due to the cumulative plastic deformation of the material at the surface after many heating and cooling cycles. Dislocations are generated and move due to stresses 
caused by thermal expansion during heating and contraction during cooling. The motion of dislocations allows persistent slip bands to form, which create extrusions and intrusions at the surface [16]. All roughness measurements were performed with a confocal microscope after 100 heating cycles. For the laser spots that melted, the depth of the melt crater dominated the roughness measurement.

Figure 6 shows that surface damage in the form of roughening is correlated with peak temperature and is independent of pulse shape. The peak surface temperature reached during the heat pulse is the most influential parameter for surface roughness compared to all other parameters we have tested. The result is consistent with [10], and for the type of W tested here, allows an approximate roughness to be predicted if the peak surface temperature is known.

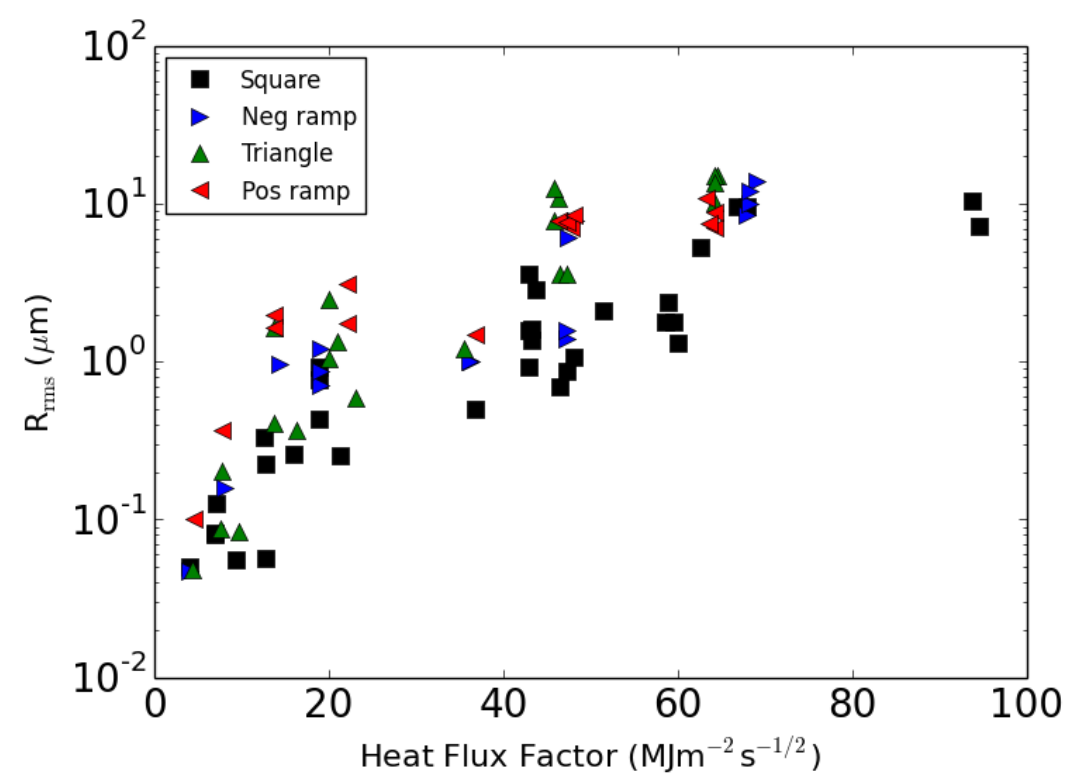

Figure 5 The root-mean-squared surface roughness $R_{r m s}$ versus the heat flux factor (defined as $F_{H F}=\frac{E}{\sqrt{\tau}}$ ) for various temporal pulse shapes. 


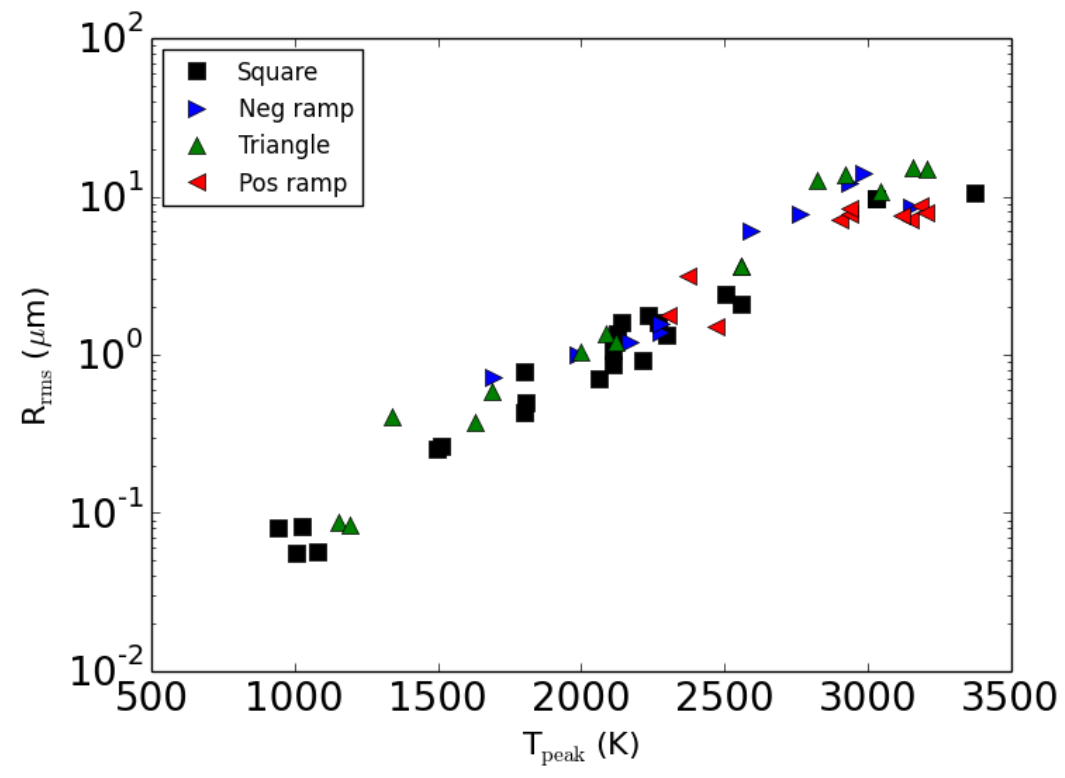

Figure 6 The root-mean-squared surface roughness $R_{r m s}$ versus the peak temperature reached during the heating pulse for various pulse shapes.

\subsection{ELM-like pulse shape and ramifications for ITER}

In order to determine whether the effect of pulse shape is beneficial or detrimental to ITER, one must understand the details of how the ITER specification for the maximum allowable ELM energy density was established. The energy density at which lanthanum tungsten castellated targets melted during QSPA plasma gun heat loading [2] was used as a basis for the ITER energy density limit for ELMs. The pulse shape of QSPA pulses was approximately a symmetric triangle with $0.5 \mathrm{~ms}$ total duration (similar to what is expected at the ITER divertor target strike points for Type I ELMs in the baseline $\mathrm{Q}_{\mathrm{DT}}=10 \mathrm{H}$-mode plasma), and the beam made an angle of 60 degrees with respect to the $\mathrm{W}$ surface normal. Melting was observed at the castellation leading edges when the energy density at the $\mathrm{W}$ surface (on the plasma stream axis) exceeded $0.4 \mathrm{MJm}^{-2}$. Due to the angle of the plasma beam, the leading edge of the castellation received approximately a factor of 2 higher heat flux than the surface, and thus the melting threshold was $\sim 0.8 \mathrm{MJm}^{-2}$ for the QSPA pulse. For ITER, the assumption was made that global top surface melting would occur on $\mathrm{W}$ if the energy density from an ELM was the same as that which caused melting from the QSPA pulse (assuming no shaping of the monoblock front surface). A margin of safety was used and the energy density limit on the $\mathrm{W}$ surface was fixed at $0.5 \mathrm{MJm}^{-2}[1,17]$. This limit also roughly corresponds to $\mathrm{W}$ melting at poloidal gaps in the tiles, assuming that the tile leading edges will receive a factor of 2 increase in heat flux. 
Since data from the QSPA test facility was used as a reference for the ITER ELM limits, we compare several experimental ELM-like pulse shapes generated with our laser to a QSPA-like pulse shape to assess the impact that a characteristic ELM pulse shape has on the ITER specification for the maximum allowable ELM energy density. The three different pulse shapes which have been tested are shown in figure 7:

1) QSPA pulse shape with duration of $0.6 \mathrm{~ms}$ (approximately a symmetric triangle, shown as the solid black lines),

2) ELM pulse shape with approximately the same energy density as the QSPA pulse (blue dashed lines), and

3) ELM pulse shape with the same peak power as the QSPA-like pulse (red dot-dashed lines).

The exact pulse shape of an ELM depends on the timescale for the collapse of the pedestal plasma at the onset of an ELM, as well as the transit time for pedestal ions to reach the divertor [18, 19]. Data for JET Type I ELMs in [4] was used to determine the characteristic ELM rise and decay times. The variability in the rise time expected for ITER ELMs is relatively low (220 - $260 \mu$ s), while the decay time has a higher degree of variability and is expected to range from approximately two to four times the rise time [4]. Confocal microscope images are used to assess material damage after 100 laser pulses, and figures 8(a), (b), and (c) correspond to the pulse shapes \#1, \#2, and \#3, respectively.
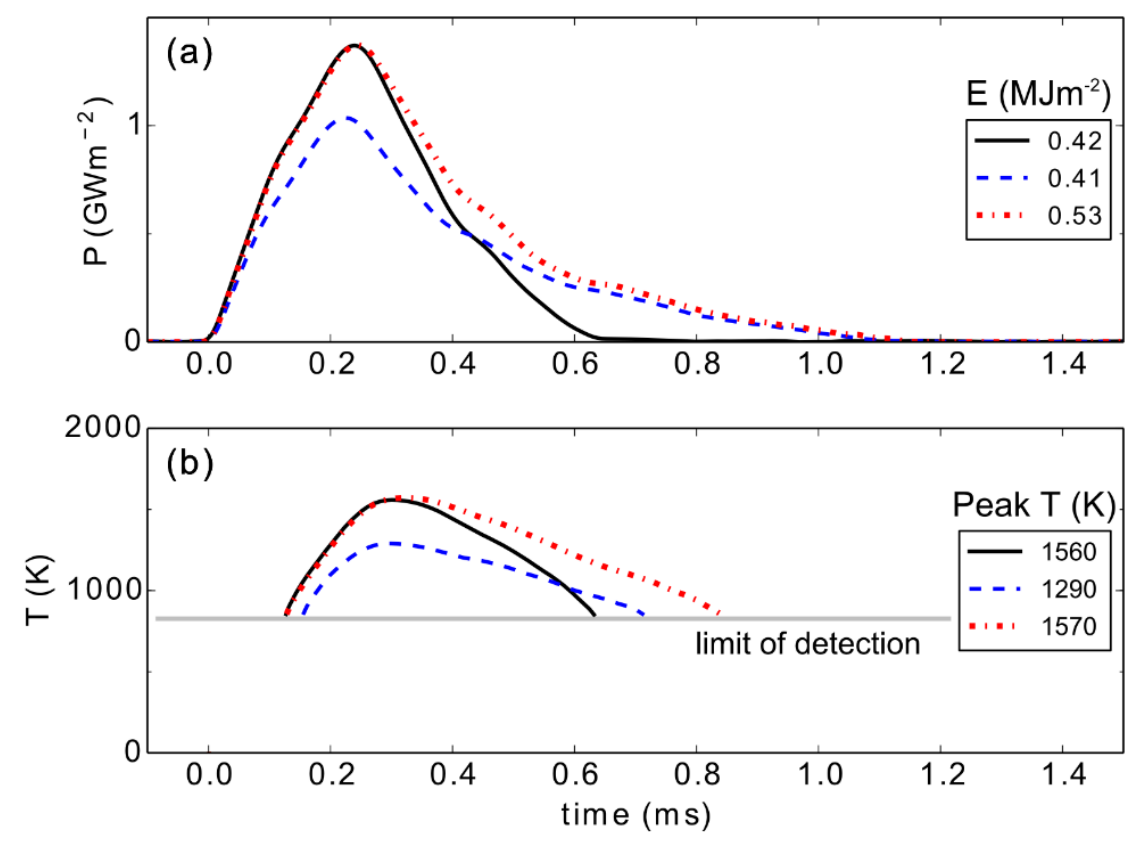

Figure 7 (a) The absorbed power density on a W surface for a QSPA-shape reference pulse (black solid line), ELMshape with the same energy density as the reference pulse (blue dashed line), and ELM shape with the same peak 
power density and rise time as the reference pulse but longer decay (red dot-dashed line). (b) The measured surface temperature from the three pulse shapes.

The peak temperature for pulse shape \#2, the ELM-like pulse with the same energy density as the reference pulse, is the lowest of the three pulse shapes. In addition, the surface roughness created by pulse shape \#2 is $R_{r m s}=0.11 \pm 0.05 \mu \mathrm{m}$, which is lower than that from the other two pulse shapes (see figure 8). This is good news for ITER because it means that there may be a margin of safety in the ELM damage threshold depending on the real ELM pulse shape compared to the reference pulse shape used to set the maximum ITER ELM energy loss. The long tail of an ELM pulse contributes to the energy density of the pulse, and normalizing the energy density to a shorter-tail reference pulse (such as QSPA) results in a smaller peak power for the ELM-like pulse.

Comparing pulse shape \#3, the ELM-like pulse with the same peak power as the reference pulse \#1, with the reference pulse itself, reveals that both the peak surface temperature and the surface roughness for the two pulse shapes are approximately the same, with the longer ELM tail causing only a small increase in both quantities. After 100 heating cycles, the ELM-like pulse \#3 created a surface roughness of $R_{r m s}=0.35 \pm 0.06 \mu \mathrm{m}$ compared to $R_{r m s}=0.30 \pm 0.05 \mu \mathrm{m}$ for the reference pulse shape. This is because the longer tail of an ELM-like pulse has only a marginal effect on raising the peak surface temperature. Thus, if a test facility is used to simulate material damage due to ELMs and is capable of creating only triangular pulses, normalizing to the peak power and ELM rise time is a more accurate method than normalizing to the energy density, because both the peak surface temperature and the resulting damage level will be a better match to those quantities expected from an ELM.

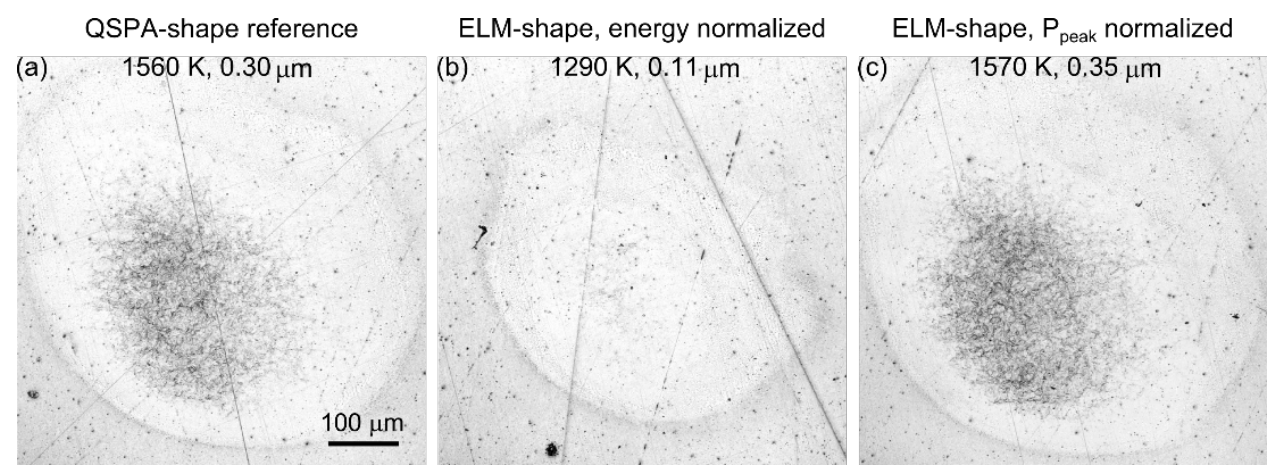

Figure 8 Confocal microscope images of laser spots after 100 heating cycles, with the peak surface temperature and $R_{r m s}$ measured near the center of the spot shown at the top of each image. (a) Laser spot using a QSPA-like reference shape, (b) ELM pulse shape with the same energy density as the reference, (c) ELM pulse shape with the same peak power as the ELM shape. The images correspond to the pulse shapes shown in figure 7. 


\section{Conclusion and outlook}

This work shows that the temporal pulse shape affects both the peak surface temperature reached during a transient heating pulse and the level of material damage. Experiments described in this paper performed in the PISCES-B facility using laser heating, as well as finite-element temperature simulations and analytic results, show that triangular-shaped pulses produce lower peak surface temperatures compared to square pulses with the same energy density and same peak power density. The peak surface temperature and resulting material damage progressively increases as the rise time increases for triangular pulses with the same energy density. The peak surface temperature reached during the pulse is the most relevant parameter for predicting surface roughness. Analytic expressions for the peak surface temperature rise allow comparisons to be made between square and triangular pulse shapes on various devices without the use of the heat flux factor, which is strictly valid only for square pulses. For non-square pulses, the pulse shape, duration, and either the peak power or the energy density of the heating pulse should be stated when reporting on transient heat experiments.

In order to assess the potential consequences for ITER, ELM pulse shapes were compared to a QSPA-like triangular pulse because the W melting threshold observed in the Russian QSPA-T plasma gun facility was used as a basis for establishing the ITER ELM energy density limit. This work shows that the long tail of an ELM has only a small effect on material damage, which should be beneficial on ITER since the real expected ELM shapes, with short rise times and longer decay times, will provide some margin relative to the established damage threshold. However, the damage threshold has been observed to decrease with larger pulse number [5], and it is currently unknown if the beneficial effects provided by the long tail of an ELM will persist at extremely high pulse number.

The majority of the data reported here was obtained at a base temperature $<325 \mathrm{~K}$, so the experiment should ideally be repeated for values above the ductile-to-brittle transition temperature. Investigating the damage response of different tungsten grades would also be useful for evaluating materials for tokamaks. In addition, future work should use much higher pulse number to determine the relative effects of temporal pulse shape and high pulse number on surface roughness development.

\section{Acknowledgements}

This work was supported by the U.S. Department of Energy, Office of Fusion Energy Sciences under Award Number DE-FG02-07ER54912. This work was also supported by The Netherlands Organisation 
for Scientific Research (NWO) within the framework of the EUROfusion Consortium and has received funding from the Euratom research and training program 2014-2018 under grant agreement number 633053. The views and opinions expressed herein do not necessarily reflect those of the European Commission or the ITER Organization.

\section{References}

1. Pitts R.A. et al 2011 J. Nucl. Mat. 415 S957-S964

2. Klimov N. et al 2009 J. Nucl. Mat. 390-391 721-726

3. Pitts R.A. et al 2013 J. Nucl. Mat. 438 S48-S56

4. Eich T. et al 2011 J. Nucl. Mat. 415 S856-S859

5. Loewenhoff Th. et al 2011 Phys. Scr. 2011014057

6. Lee W.S. et al 1998 Mat. Science and Eng. A 247 102-112

7. Doerner R.P. et al 2004 Phys. Scr. T111 75

8. Yu J.H. et al 2013 J. Nucl. Mat. 438 S1150

9. Sakaguchi W. et al 2009 J. Nucl. Mat. 3901149

10. Van Eden G.G. et al 2014 Nucl. Fusion 54123010

11. Bäuerle D.W. 2011 Laser Processing and Chemistry $4^{\text {th }}$ edn (Berlin: Springer)

12. Yu J.H. et al 2014 Phys. Scr. T159 014036

13. Morgan T.W. et al 2013 J. Nucl. Mat. 438 S96-101

14. Suslova S. et al 2015 Scientific Reports 46845

15. Linke J. et al 2007 J. Nucl. Mat. 367-370 1422-1431

16. Suresh S. 2003 Fatigue of Materials $2^{\text {nd }}$ edn (Cambridge: Cambridge University Press)

17. Loarte A. et al 2014 Nucl. Fusion 54033007

18. Yu J.H. et al 2008 Phys. Plasmas 15032504

19. Loarte A. et al 2003 Plasma Phys. Control. Fusion 45 1549-1569 